\title{
Assessing different measures of population-level vaccine protection using a case-control study
}

Mohammad $\mathrm{Ali}^{1,2}$, Young Ae You ${ }^{1}$, Suman Kanungo ${ }^{3}$, Byomkesh Manna ${ }^{3}$, Jacqueline L. Deen ${ }^{4}$,

Anna Lena Lopez ${ }^{5}$, Thomas F Wierzba ${ }^{1}$, Sujit K Bhattacharya ${ }^{3}$, Dipika Sur ${ }^{3}$, John D Clemens ${ }^{6,7}$

\section{Running title: Population-level vaccine protection}

${ }^{1}$ International Vaccine Institute, Seoul, Republic of Korea

2Johns Hopkins Bloomberg School of Public Health, Baltimore, USA

${ }^{3}$ National Institute of Cholera and Enteric Diseases, Kolkata, India

${ }^{4}$ Menzies School of Health Research, Casuarina, NT, Australia

${ }^{5}$ University of the Philippines Manila, National Institutes of Health

${ }^{6}$ icddr,b, Dhaka, Bangladesh

${ }^{7}$ UCLA Fielding School of Public Health, Los Angeles, USA

\section{Corresponding author}

Mohammad Ali, PhD

Department of International Health

Johns Hopkins Bloomberg School of Public Health

615 N.Wolfe Street / E5543

Baltimore, Maryland 21205

410-502-3154 (office)

410-502-6733 (fax)

Email:mali25@jhu.edu 


\section{Abstract}

Background: Case-control studies have not been examined for their utility in assessing population-level vaccine protection in individually randomized trials.

Methods: We used the data of a randomized, placebo-controlled trial of a cholera vaccine to compare the results of case-control analyses with those of cohort analyses. Cases of cholera were selected from the trial population followed for three years following dosing. For each case, we selected 4 age-matched controls who had not developed cholera. For each case and control, GIS was used to calculate vaccine coverage of individuals in a surrounding "virtual" cluster. Specific selection strategies were used to evaluate the vaccine protective effects. Results: 66,900 out of 108,389 individuals received two doses of the assigned regimen. For direct protection among subjects in low vaccine coverage clusters, we observed $78 \%$ (95\% Cl:47\%-91\%) protection in a cohort analysis and $84 \%$ (95\% Cl:60\%-94\%) in case-control analysis after adjusting for confounding factors. Using our GIS-based approach, estimated indirect protection was $52 \%(95 \% \mathrm{Cl}: 10 \%-74 \%)$ in cohort and $76 \%$ (95\% Cl:47\%-89\%) in case control analysis. Estimates of total and overall effectiveness were similar for cohort and casecontrol analyses.

Conclusion: The findings show that case-control analyses of individually randomized vaccine trials may be used to evaluate direct as well as population-level vaccine protection.

Key Words: Vaccine effectiveness; herd effect; case-control study; cohort study; randomized controlled trial 


\section{BACKGROUND}

Studies of vaccine protection that include assessments of population-level (herd) vaccine protective effects may be useful for informing policy decisions by increasing our understanding of vaccine impact and cost-effectiveness [1]. Moreover, demonstration of the herd protective effects for vaccines conferring only a moderate level of direct protection may strengthen the case for introducing such vaccines into public health practice [2].

Cluster-randomized, placebo-controlled trials, where households or geographic regions commonly serve as clusters, have been proposed as ideal for evaluating the different types of herd protective effects of a vaccine $[3,4]$, and such trials have been successfully used to achieve this purpose [5]. However, cluster-randomized trials are expensive and logistically demanding, and it is not always possible to identify epidemiologically suitable clusters as units of randomization [1].

Recently, we have demonstrated that evaluation of vaccine herd protective effects may also be possible with individually randomized trials and with observational cohort studies, making use of geographic information systems (GIS) to create virtual clusters of people in conjunction with epidemiological data [6-8]. In principle, assessment of vaccine herd protection can also be accomplished with case-control studies, either in circumstances in which the vaccine has been given in a randomized trial or in those in which vaccine is administered according to routine practice. However, case-control analyses have rarely been used for this purpose, perhaps because a framework for evaluating the different kinds of vaccine herd protective effects has not been clearly outlined for case-control studies. This omission may be important, because casecontrol studies are known to confer the potential advantages of increased statistical efficiency, lower expense, and rapid execution [9]. Herein, we describe how case-control analyses can be applied to evaluate different population-level protective effects of a vaccine in an individually 
randomized, placebo-controlled trial, illustrating the approach with a recently completed field trial of a killed oral cholera vaccine in Kolkata, India.

\section{METHODS}

\section{Estimation of vaccine protective effects in a cluster randomized trial}

Conventional evaluations of vaccine protection attempt to measure direct vaccine protection: individual protection of a vaccinated person in isolation from vaccine protection of surrounding persons. Direct protection is due solely to the elicitation of relevant immune responses by the vaccine in the vaccine recipient. In contrast, an evaluation of population level protective effects of vaccination requires consideration of both the direct protective effects of a vaccine as well as vaccine herd protective effects above and beyond direct protection. Herd protective effects are conventionally classified as indirect vaccine protection-protection of nonvaccinees due to vaccination of their neighbors or contacts; total vaccine protection-protection of vaccinees due to direct effects and added protection of vaccinees owing to vaccination of their neighbors or contacts; and overall protection-protection of an entire population, irrespective of the vaccination status of its individual members, due to the combination of total and indirect protection. Another measurement, enhanced vaccine protection, can be defined as the extent to which total vaccine protection increases with increasing vaccine coverage.

In a cluster randomized trial in which clusters of persons are allocated to vaccine or placebo, direct protection is estimated by comparison of the incidence of the target disease in vaccinees versus non-vaccinees in the vaccine clusters; indirect protection by the comparison of incidence in non-dosed individuals in vaccine versus placebo clusters; total protection by the comparison of incidence in vaccinees in vaccine clusters versus placebo recipients in placebo clusters; overall protection by the comparison of incidence among all persons in vaccine versus placebo clusters; and enhanced protection by comparison of incidence in vaccinees with increasing levels of vaccine coverage in their clusters. 


\section{Estimation of vaccine protective effects using cohort approach in an individually randomized trial}

In a cohort analysis of a placebo-controlled, individually randomized trial, with virtual clusters around each individual constructed by a GIS and vaccine coverage computed for each cluster, direct protection is estimated by comparing the incidence of the target disease in vaccinees and placebo recipients, ideally those in low vaccine coverage clusters, for reasons discussed elsewhere [6]. To estimate population-level vaccine protective effects, use is made of the level of vaccine coverage of the virtual clusters created by the GIS strategy, in which constituents are admixtures of vaccinees and placebo recipients and non-participants. In this approach, indirect protection is estimated by comparing the incidence in placebo recipients in clusters with lowest levels of vaccine coverage versus with those in higher coverage clusters; total protection is estimated by comparing the incidence in vaccinees in the lowest vaccine coverage clusters versus placebo recipients in higher vaccine coverage clusters; overall protection is estimated by comparing the incidence among all individuals in the lowest coverage clusters versus all those in higher vaccine coverage clusters; and enhanced protection is estimated by comparing the incidence in vaccinees in higher versus lowest vaccine coverage clusters $[6,7]$. Such cohort analyses can also be done for situations in which vaccines are given in routine practice in a non-randomized fashion, though in the absence of a placebo, comparisons must be done with groups of non-vaccinated persons rather than placebo recipients [8].

\section{Evaluation of vaccine protective effects using case-control analysis in an individually randomized trial}

In this approach, incident cases with the disease are compared with non-disease controls selected from a population of non-cases who would have been detected as cases had they developed the disease. As in the cohort analyses, GIS is used to construct a virtual cluster 
and vaccine coverage is computed for each cluster. In contrast to the cohort analyses, the clusters are created around each selected case and control, rather than all individuals in the population. Because odds ratios derived from appropriately designed case control studies estimate risk ratios (or rate ratios) from corresponding cohort studies, case control analyses can estimate risk ratios (or rate ratios) and thus levels of protection for the corresponding cohort analyses [9]. As Figure 1 shows, the underlying cohort analyses of an individually randomized placebo-controlled vaccine trial thereby guide the selection of cases and controls, as well as definitions of vaccination exposures, to estimate the different types of vaccine protection in case-control analyses.

Direct protection is estimated from odds ratios relating individual level vaccination status to case-control status among cases and controls who have received vaccine or placebo, ideally among individuals in low vaccine coverage clusters [6]. Population-level vaccine protective effects are measured by odds ratios relating vaccine coverage of the cluster of residence (lowest versus higher) to case-control status, where cases and controls are defined differently depending on the measure of protection. Indirect vaccine protection is measured among cases and controls who have received placebo; total protection is evaluated among cases and controls who have received placebo in the lowest vaccine coverage clusters or vaccine in higher vaccine coverage clusters; overall protection is measured among all cases and controls, regardless of the agent received; and enhanced protection is assessed among vaccinated cases and controls.

\section{Application of the cohort and case-control approaches to a cholera vaccine trial in}

\section{Kolkata}

The trial

We illustrate the cohort and case-control approaches by analysis of a randomized trial of a cholera vaccine conducted in three wards (29, 30 and 33) of Kolkata, India, with a population 
of about 109,000 residing in 3,933 dwellings. Details of the study design and procedures are described elsewhere [10,11]. In brief, a census of the population with geographic coordinates of each household was done at baseline. Eligible residents were randomized, by dwelling, to receive a two-dose regimen of either oral cholera vaccine (OCV) or oral placebo. Residents aged one year and older who were not pregnant were invited to participate in the vaccination campaign. Administration of vaccine and placebo was undertaken in two rounds between July 27 and September 10, 2006. Surveillance for cholera was conducted in nine project health clinics and two government hospitals. For the present analysis, we focused on cholera occurring during the first three years of surveillance after dosing of subjects. A cholera case was defined as a non-bloody diarrheal episode in which Vibrio cholerae $\mathrm{O} 1$ was isolated from the fecal specimen, and a domiciliary visit to the individual's residence confirmed that the identified person had in fact sought care for diarrhea on the date of presentation. Because we did not detect a design effect owing to randomization by dwelling and because we did not observe vaccine herd effects when analyzed as a cluster-randomized trial with dwellings as clusters, we conducted cohort and case-control analyses of the trial as if the study had been an individually randomized, placebo-controlled trial. Several of the cohort analyses are reported in earlier papers [10].

\section{Assessment of vaccine coverage of clusters}

In both the cohort and case-control analyses of population-level herd effects, it was necessary to calculate vaccine coverage for the cluster surrounding each individual. To define the size of the cluster surrounding each individual, we investigated different scales with the use of statistical criteria that were independent of analyses of vaccine protective effects and identified a radius around the household of residence of $250 \mathrm{~m}$ as the optimum size [7]. The cluster level vaccine coverage surrounding each person was calculated as the number of vaccinated individuals (two-dose recipients) divided by the number of persons who were age- 
eligible for the trial residing within $250 \mathrm{~m}$ radius of the individual's residence at zero time. Zero time was defined as the date of the second dose for 2-dose recipients of vaccine or placebo, and as the median date of $2^{\text {nd }}$ dose persons receiving 2-doses in the cluster for persons receiving one or no dose.

\section{Analysis of vaccine protective effects in cohort analyses}

As presented elsewhere [11], we assessed all vaccine protective effects from zero time until 1095 days later. Only initial cholera episodes with onsets at least 14 days after zero time were counted. Vaccine protection was calculated as (1- relative rate of cholera in the compared exposure categories) $\times 100 \%$. Direct vaccine protection was measured by contrasting the incidence of cholera in persons who had received 2 doses of vaccine versus those who had received two doses of placebo. Indirect vaccine protection was evaluated by comparing the incidence in 2-dose placebo recipients residing in clusters with the highest versus lowest quintiles of vaccine coverage. We assessed total vaccine protection by contrasting the incidence in 2-dose vaccinees residing in the highest quintile vaccine coverage clusters versus 2-dose placebo recipients in the lowest quintile coverage clusters, and enhanced vaccine protection by comparing the incidence in 2-dose vaccinees living in the highest versus lowest quintile clusters.

To assess overall vaccine protection, we contrasted the incidence among all persons (including persons who were ineligible to receive the vaccine) in the highest versus lowest quintile coverage clusters. To adjust for potential confounding variables, we used Cox regression models, verifying first that the proportionality assumption was fulfilled and exponentiating the dichotomous principal exposure variable (vaccination or vaccine coverage stratum) to estimate vaccine protection, taking the standard error of this coefficient to estimate P-values and 95\% confidence intervals. Potential confounding variables, such as age at zero time, household ownership of at least one luxury item-a proxy for higher socioeconomic status, 
and distance from the household to the nearest health clinic, were considered for inclusion as independent variables in these models. To avoid overfitting the models, we used a backward elimination algorithm to select independent variables related to the time to cholera at $p<0.10$. In all models, we used age, owning at least one luxury item, and distance from the household to the nearest health clinic, which are usually found to be associated with the risk for cholera. All P values and $95 \%$ confidence intervals were 2 -tailed.

Analysis of vaccine protective effects in case-control analyses

Cases and controls were selected by incidence density sampling. Both cases and controls were residents of the study area at zero time. Cases were first episodes of cholera detected from 14 to 1095 days after zero time. For each case, we selected at random four controls who had not developed cholera up until the time of onset of cholera in the matched case. Controls were matched to cases by age at zero time ( $<5$ years; $5-14$ years; 15 years and older), as these age strata are cogent determinants of the risk of cholera in the Kolkata setting. Vaccine protection was calculated as (1- odds ratio relating case-control status to the compared exposure categories) $\times 100 \%$. We assessed direct protection by assessing cases and controls who had received either two doses of vaccine or placebo. Indirect, total, and enhanced vaccine protection were evaluated as described above among cases and controls who had received 2doses of vaccine and/or placebo, and overall protection was assessed among all cases and controls irrespective of whether they had received vaccine or placebo. In each of these analyses of population-level vaccine protection, cases and controls were contrasted for whether they resided in clusters in the highest versus the lowest quintiles of 2-dose vaccine coverage. Crude odds ratios were statistically evaluated with the chi-square test, or with Fisher's exact test when mandated by small numbers. Adjusted odds ratios were estimated in logistic regression models, with case-control status as the dependent variable and the main dichotomous exposure variable (vaccinated or vaccine coverage stratum) and the same confounding variables as used in the 
cohort analysis as the independent variables. We used conditional logistic regression for the case-control analysis when the entire study set was analyzed. However, due to the drop out of multiple cases and controls when were analyzed, due to breakage of matched case control sets, we used unconditional logistic regression for analyses within subgroups, controlling for the matching variables and other covariates associated with the cholera outcome. As in the cohort models, the coefficient for the main exposure variable was exponentiated to estimate adjusted odds ratios and standard errors of these coefficients were used to estimate $P$ values and $95 \%$ confidence intervals. Selection of covariates for these models and use of 2-tailed statistical tests mirrored the cohort analyses.

\section{Ethical considerations}

Informed consent was obtained, as described elsewhere [5]. The original protocol was approved by the ethics committee of the National Institute of Cholera and Enteric Diseases (NICED), the Health Ministry Screening Committee of India, and the International Vaccine Institute Institutional Review Board. The trial was registered at ClinicalTrials.gov, number NCT00289224.

\section{RESULTS}

There were 108,349 individuals in the study population and of them 66,900 persons received 2-dose of the regimen assigned for them. Using conventional analyses to assess direct protection (Table 1), the point estimate of direct vaccine protection was slightly higher in the case-control analysis $(76 \%$; $95 \% \mathrm{Cl}: 63 \%-8 \%$; $p<.01)$ than in the cohort analysis $(66 \% ; 95 \% \mathrm{Cl}: 51 \%-76 \% ; \mathrm{p}<.01)$ after adjusting for the potential confounders. However, the point estimates fell within the $95 \% \mathrm{Cls}$ of one another. In both cohort and case-control analyses, direct protection rose notably as the level of cluster level vaccine coverage fell, being $78 \%(95 \% \mathrm{Cl}: 47 \%-91 \%, \mathrm{p}<.01)$ in the cohort analysis and 
$84 \%(95 \% \mathrm{Cl}: 60 \%-94 \%, \mathrm{p}<.01)$ in the case-control analysis of individuals residing in clusters with $\leq 25 \%$ coverage. After adjustment for potential confounders, direct vaccine protection varied inversely with vaccine coverage in both cohort $(p=.02)$ and case-control $(p<.01)$ analyses. However, in contrast to the cohort analysis, in which protection was notably higher in the $5^{\text {th }}$ than the $4^{\text {th }}$ lowest quintiles of vaccine coverage, the case control analysis failed to show this pattern.

To assess population-level vaccine protective effects we selected as a referent the lowest quintile of vaccine coverage, $25 \%$ or lower, and contrasted this category of coverage with the highest quintile of vaccine coverage, $34 \%$ or more. The evaluation of indirect vaccine protection yielded $52 \%$ protection $(95 \% \mathrm{Cl}: 10 \%-74 \% ; \mathrm{p}=.02)$ in the cohort analysis and $76 \%$ (95\% Cl: 47\%-89\%; $p=<.01)$ in the case-control analysis. Total protection was $70 \%(95 \%$ $\mathrm{Cl}: 40 \%-85 \%, \mathrm{p}=<.01)$ in the cohort and $75 \%(95 \% \mathrm{Cl}: 43 \%-89 \%, \mathrm{p}<.01$ in the case-control analysis. And, vaccine overall protection was 44\% (95\% Cl:11\%-68\%, p=.01) in the cohort and $44 \%(95 \% \mathrm{Cl}:-7 \%-70 \%, p=.08)$ in the case-control analysis. The results of the analysis for the enhanced protection did not yield evidence of this type of protection in either the cohort or casecontrol analyses (Table 2).

\section{DISCUSSION}

The GIS-based case-control approach for estimating population-level effectiveness reported on here yielded results in the Kolkata OCV trial similar to those derived using our previously reported GIS-based cohort approach [7]. Our study delineates how case-control analyses can be used to estimate different measures of vaccine herd protection in individually randomized vaccine trials. In one previously reported study [12], the vaccine herd protective effect of a Haemophilus influenzae type b (Hib) conjugate vaccine was assessed comparing the level of Hib vaccine coverage of neighborhoods of cases of invasive Hib disease with that for controls, selected without regard to vaccination status. The study thereby assessed overall vaccine protection, and, like the analyses conducted in the present paper, found evidence of 
this type of protection. No analyses of indirect, total, or enhanced protection were reported. Our analysis of the different types of population-level vaccine protection measurable in case-control studies also illustrates the importance of specifying the type of vaccine protection that is being addressed.

A striking feature of the cohort and case-control analyses of the Kolkata trial was the observation of an inverse relationship between vaccine coverage of the cluster and estimated direct vaccine protection, suggesting that, for some vaccines, conventional estimates of direct vaccine protection may be distorted by the population herd effects of vaccine coverage. This relationship was also observed earlier in a field trial of OCVs in Bangladesh [13]. There was a discrepancy between the cohort and case control analyses in that direct protection fell in the $5^{\text {th }}$ lowest quintile of vaccine coverage in the former but not the latter. Whether this apparent discrepancy was real or due to chance cannot be ascertained with certainty, as the point estimates for vaccine protection in the $5^{\text {th }}$ lowest quintiles for each analysis fell within the $95 \%$ $\mathrm{Cl}$ of the other analysis. Given the consistency of this observed inverse relationship between measured direct vaccine protection and local vaccine coverage, this observation underscores the need to consider vaccine coverage, often using GIS, even in conventional cohort and casecontrol analyses of direct vaccine protection. It also contradicts the assertion that conventional estimates of vaccine protection in case-control studies are not affected by vaccine coverage of the population [9]. And, the observation suggests that differences in vaccine coverage might explain some of the variability in reported levels of direct protection conferred by killed OCVs in different settings $[14,15]$.

It might be argued that our use of the Kolkata dataset presented an insufficient challenge for an assessment of the validity of the case-control approach, as this was a placebo-controlled, randomized trial and the use of both randomization and double-blinding minimized many of the biases to which case-control analyses of non-experimental study designs are vulnerable. For example, the restriction of analyses of indirect protection to placebo recipients presents distinct 
methodological advantages, as it is well known that placebo recipients differ in many ways from other non-vaccinees, some of which may influence the risk of cholera [16]. In studies lacking a placebo, we propose the use of analyses of "bias indicator conditions", disease outcomes similar to the primary outcome under analysis (in this case, cholera), but unlikely to prevented by vaccine direct or herd effects, for signaling whether bias was likely to have occurred. Using this approach we have previously shown that substitution of all non-vaccinees for placebo recipients yielded apparently unbiased estimates of vaccine herd protection in a cholera vaccine demonstration project in Zanzibar [8] Another limitation of our case-control analysis is that it made use of vaccine coverage data at baseline, which may not be available in studies unless planned prospectively, and did not account for the changing levels of vaccine coverage over time, due to population movements.

As well, several intrinsic limitations of the case-control approach for evaluating the different measures of vaccine effectiveness require comment. First, a weakness shared by all studies that do not use the cluster-randomized paradigm is that they do not permit use of nonvaccinated control clusters for estimation of protection, but rely on use of low vaccine coverage clusters as a referent, possibly underestimating total, indirect, and overall vaccine protection. Second, the GIS-based case-control approach is more difficult to understand than our earlier reported GIS-based cohort analyses. Third, similar to cohort studies, this approach also requires a GIS for creation of clusters to estimate vaccine coverage levels of clusters around analyzed individuals. If such clusters are not based on a GIS-based census at baseline, which may occur in a circumstances in which case-control design might be considered, retrospective reconstruction of clusters and coverage levels will be necessary, which may or may not be feasible. Another critical issue shared by both the GIS-based cohort and case-control analyses, which entail "virtual" reconstruction of clusters around individuals for estimating vaccine coverage, is the choice of a method for selection of an appropriate cluster size. The cluster size for the analyses reported in this paper was based on a statistical method that selected the size 
independently of estimates of population-level vaccine protection, as described in our earlier work [7], but more methodological work in this area is needed.

Nonetheless, case-control studies of vaccine protection may offer the advantages of statistical efficiency, logistical and financial economy, and the ability to produce results based on actual field conditions in a relatively short time frame [17]. The approach outlined here, for example, might be used when GIS is not planned or implemented at baseline in a vaccine trial or program, and evaluation of vaccine herd effects is an objective. Focusing a GIS assessment only on cases and controls found in an existing baseline census of the study population of interest, rather than on all members of the census, may permit a feasible approach to assessing these effects.

\section{Funding}

No funding was sought for conducting this study.

\section{Acknowledgement}

We are grateful to the people of the study area in Kolkata and the field staff for whom the project was successfully conducted. We thank to the Bill \& Melinda Gates Foundation for supporting the project from which the data were collected to conduct this study.

\section{Author Contributions}

MA and JDC contributed to the study design. MA, SK, BM, JLD, ALP, LVS, TFW, SKB, DS, and JDC contributed to the implementation and supervision of the study. MA, YAY, and JDC analyzed the data and take responsibility for the accuracy of the data analysis. All authors participated in the writing of the manuscript and had full access to the data in the study. All authors saw and approved the final version of the manuscript. 


\section{References}

[1] Clemens J, Shin S, Ali M. New approaches to the assessment of vaccine herd protection in trials conducted before licensure. Lancet Infectious Diseases 2011;11(6):482-7.

[2] Longini I, Nizam A, Ali M, Yunus M, Shenvi N, Clemens J. Controlling endemic cholera with oral vaccines. PLoS Med 2007; 4: e336.

[3] Halloran ME, Struchiner CJ, Longini IM Jr. Study designs for evaluating different efficacy and effectiveness aspects of vaccines. Am J Epidemiol 1997; 146:789-803.

[4] Clemens J, Brenner R, Rao M, Tafari N, Lowe C. Evaluating new vaccines for developing countries. Efficacy or effectiveness? JAMA 1996;275(5):390-7.

[5] Sur D, Ochiai RL, Bhattacharya SK, Nirmal K. Ganguly NK, Ali M, et al. A cluster-randomized effectiveness trial of Vi typhoid vaccine in India. N Engl J Med 2009; 361: 335-44.

[6] Ali M, Emch M, von Seidlein L, Yunus M, Sack DA, Rao M, et al. Herd immunity conferred by killed oral cholera vaccines in Bangladesh: a reanalysis. Lancet 2005; 366:44-9.

[7] Ali M, Sur D, You YA, Kanungo S, Sah B, Manna B, et al. Herd protection by a bivalentkilled-whole-cell oral cholera vaccine in the slums of Kolkata, India. Clinical Infectious Diseases 2013;56(8):1123-31.

[8] Khatib AM, Ali M, von Seidlein L, Kim DR, Hashim R, Reyburn R, et al. Effectiveness of an oral cholera vaccine in Zanzibar: findings from a mass vaccination campaign and observational cohort study. Lancet Infectious Diseases 2012;12(11):837-844.

[9] Rodrigues LC, Smith PG. Use of the case-control approach in vaccine evaluation: efficacy and adverse effects. Epidemiological Reviews 1999; 21(1):56-72.

[10] Sur D, Lopez AL, Kanungo S, Paisley A, Manna B, Ali M, et al. Efficacy and safety of a modified killed-whole-cell oral cholera vaccine in India: an interim analysis of a clusterrandomised, double-blind, placebo-controlled trial. Lancet 2009; 374:1694-1702. 
[11] Sur D, Kanungo S, Sah B, Manna B, Ali M, paisley AM, et al. Efficacy of a low-cost, killed whole-cell oral cholera vaccine: results from 3 years of follow up of a cluster-randomized, placebo-controlled trial. PLoS Neglected Tropical Diseases 2011; 5(10):e1289.

[12] Chen WJ, Moulton LH, Saha SK, Mahmud AA, Arifeen SE, Baqui AH. Estimation of the herd protection of Haemophilus influenzae type b conjugate vaccine against radiologically confirmed pneumonia in children under 2 years old in Dhaka, Bangladesh. Vaccine 2014;32(8):944-8.

[13] Ali M, Emch M, von Seidlein L, Yunus M, Sack DA, Rao M, et al. Herd immunity conferred by killed oral cholera vaccines in Bangladesh: a reanalysis. Lancet 2005; 366:44-9.

[14] Luquero FJ, Grout L, Ciglenecki I, Sakoba K, Traore B, Heile M, et al. Use of Vibrio cholerae vaccine in an outbreak in Guinea. N Engl J Med 2014;370(22):2111-20.

[15] Lucas M, Deen JL, von Seidlein L, Wang XY, Ampuero J. Puri M, et al. High-level effectiveness of a mass oral cholera vaccination in Beira, Mozambique. The New England Journal of Medicine 2005; 352(8):757-67.

[16] Clemens JD, van Loon FF, Rao M, Ahmed F, Chakraborty J, Khan MR, et al. Nonparticipation as a determinant of adverse health outcomes in a field trial of oral cholera vaccines. American Journal of Epidemiology 1992; 135:865-874.

[17] Comstock GW. Evaluating vaccination effectiveness and vaccine efficacy by means of case-control Studies. Epidemiologic Reviews 1994; 16(1):77-89 
Figure 1. Selection of cases and controls and definition of vaccine exposure from underlying cohorts of an individually randomized placebo-controlled vaccine trials

Type of protection Source of population exposure

Direct $\longrightarrow$ Vaccinees and placebo

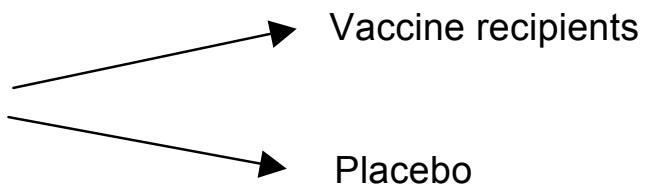

Indirect $\longrightarrow$ Placebo recipients

Total $\longrightarrow$ Vaccinees and placebo
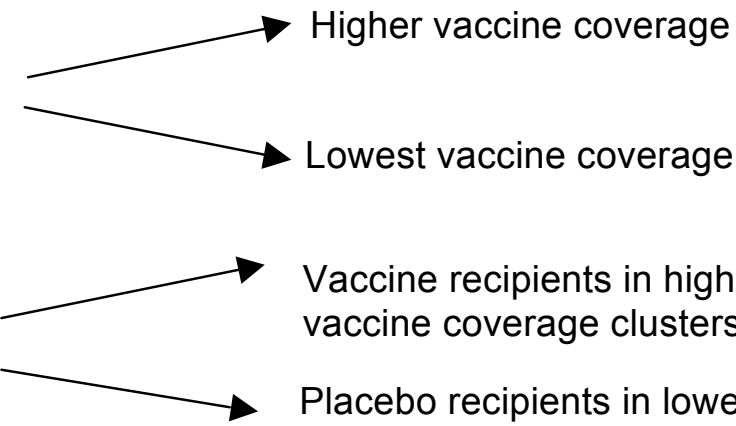

Vaccine recipients in higher vaccine coverage clusters

Placebo recipients in lowest vaccine coverage clusters

Enhanced $\longrightarrow$ Vaccinees

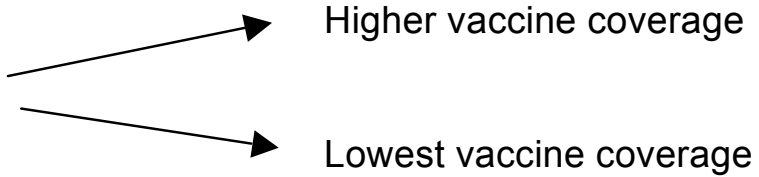

Overall Vaccinees, placebo recipients and non-dosed individuals

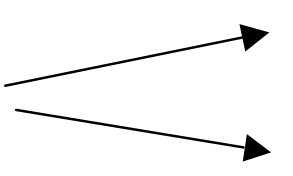

All individuals in higher vaccine coverage clusters

All individuals in lowest vaccine coverage clusters 
Table 1. Direct vaccine protection by cluster level of coverage in cohort and case-control analyses*

\begin{tabular}{|c|c|c|c|c|c|c|c|c|c|c|c|c|c|c|c|c|c|}
\hline \multirow{3}{*}{$\begin{array}{l}\text { Cluster leve } \\
\text { of coverage } \\
(\%)\end{array}$} & \multirow{3}{*}{$\begin{array}{c}\text { Populat } \\
\text { ion }\end{array}$} & \multirow{2}{*}{\multicolumn{2}{|c|}{ Cases }} & \multirow{2}{*}{\multicolumn{2}{|c|}{ Controls }} & \multicolumn{6}{|c|}{ Cohort analysis $^{\dagger}$} & \multicolumn{6}{|c|}{ Case-control analysis $^{\ddagger}$} \\
\hline & & & & & & \multicolumn{3}{|c|}{ Crude } & \multicolumn{3}{|c|}{ Adjusted $^{\S}$} & \multicolumn{3}{|c|}{ Crude } & \multicolumn{3}{|c|}{ Adjusted $^{\S}$} \\
\hline & & Vaccine & Placebo & Vaccine & Placebo & $\begin{array}{l}\text { VE } \\
(\%)\end{array}$ & $95 \% \mathrm{Cl}$ & $p$ & $\begin{array}{l}\text { VE } \\
(\%)\end{array}$ & $95 \% \mathrm{Cl}$ & $\mathrm{P}$ & $\begin{array}{l}\text { VE } \\
(\%)\end{array}$ & $95 \% \mathrm{Cl}$ & $\mathrm{p}$ & $\begin{array}{l}\text { VE } \\
(\%)\end{array}$ & $95 \% \mathrm{Cl}$ & $\mathrm{P}$ \\
\hline $0.00-25.00$ & 11,906 & 6 & 40 & 54 & 81 & 77 & $46-90$ & $<.01$ & 78 & $47-91$ & $<.01$ & 78 & $43-91$ & $<.01$ & 84 & $60-94$ & $<.01$ \\
\hline $25.01-28.00$ & 13,891 & 9 & 44 & 53 & 29 & 74 & $46-87$ & $<.01$ & 73 & $46-87$ & $<.01$ & 89 & $74-95$ & $<.01$ & 92 & $76-97$ & $<.01$ \\
\hline $28.01-31.00$ & 12,943 & 7 & 15 & 44 & 51 & 59 & $0-83$ & .05 & 60 & $2-84$ & .04 & 46 & $-45-80$ & 0.22 & 43 & $-53-79$ & .26 \\
\hline $31.01-34.00$ & 12,870 & 6 & 15 & 68 & 74 & 57 & $-11-83$ & .08 & 58 & $-9-84$ & .07 & 56 & $\begin{array}{l}-19-84 \\
\end{array}$ & 0.10 & 55 & $-25-84$ & .13 \\
\hline$\geq 34.01$ & 15,290 & 10 & 14 & 139 & 71 & 36 & $-45-71$ & .29 & 36 & $-45-72$ & .29 & 63 & $14-85$ & 0.02 & 68 & $22-87$ & .01 \\
\hline \begin{tabular}{|l|} 
All \\
individuals
\end{tabular} & 66,900 & 38 & 128 & 358 & 306 & 68 & $53-77$ & $<.01$ & 66 & $51-76$ & $<.01$ & 75 & $62-83$ & $<.01$ & 76 & $63-84$ & $<.01$ \\
\hline
\end{tabular}

*Vaccine means vaccine recipients and placebo means placebo recipients.

${ }^{\dagger}$ Cox proportional hazard model was used.

¥ Unconditional logistic model was used for the individual strata and conditional logistic model was used for all individuals.

$\S$ Variables selected as covariates were age at $2^{\text {nd }}$ dose, individuals living in a household owning at least one luxury item, distance from the household to the nearest health clinic for first and second quintile and for all individuals; age at $2^{\text {nd }}$ dose for the third, fourth, and fifth quintiles. 
Table 2. Different measures of population-level vaccine protection in cohort and case-control analyses*

\begin{tabular}{|c|c|c|c|c|c|c|c|c|c|c|c|c|c|c|c|c|c|}
\hline \multirow{3}{*}{$\begin{array}{l}\text { Measure } \\
\text { of } \\
\text { protection }\end{array}$} & \multirow{2}{*}{\multicolumn{2}{|c|}{$\begin{array}{c}\text { Population } 1 \\
\text { (P1) }\end{array}$}} & \multirow{2}{*}{\multicolumn{2}{|c|}{$\begin{array}{l}\text { Population } 2 \\
\text { (P2) }\end{array}$}} & \multirow{3}{*}{$\begin{array}{c}\text { No. of } \\
\text { controls }\end{array}$} & \multicolumn{6}{|c|}{ Cohort analysis $^{\dagger}$} & \multicolumn{6}{|c|}{ Case-control analysis ${ }^{\ddagger}$} \\
\hline & & & & & & \multicolumn{3}{|c|}{ Crude } & \multicolumn{3}{|c|}{ Adjusted $^{\S}$} & \multicolumn{3}{|c|}{\begin{tabular}{l|l} 
Crude & \\
\end{tabular}} & \multicolumn{3}{|c|}{ Adjusted $^{\S}$} \\
\hline & $\begin{array}{c}\text { No. of } \\
\text { subject } \\
\text { s }\end{array}$ & $\begin{array}{l}\text { No. of } \\
\text { cases }\end{array}$ & $\begin{array}{l}\text { No. of } \\
\text { subjects }\end{array}$ & $\begin{array}{l}\text { No. of } \\
\text { cases }\end{array}$ & & $\begin{array}{l}\text { VE } \\
(\%)\end{array}$ & $95 \% \mathrm{Cl}$ & $\mathrm{p}$ & $\begin{array}{l}\text { VE } \\
(\%)\end{array}$ & $95 \% \mathrm{Cl}$ & $\mathrm{P}$ & $\begin{array}{l}\text { VE } \\
(\%)\end{array}$ & $95 \% \mathrm{Cl}$ & $\mathrm{p}$ & $\begin{array}{l}\text { VE } \\
(\%)\end{array}$ & $95 \% \mathrm{Cl}$ & $P$ \\
\hline Indirect & 7,244 & 14 & 7,219 & 40 & 216 & 66 & $38-82$ & $<.01$ & 52 & $10-74$ & 0.02 & 76 & $53-88$ & $<.0001$ & 76 & $47-89$ & $<.01$ \\
\hline Total & 8,046 & 10 & 7,219 & 40 & 200 & 78 & $56-89$ & $<.01$ & 70 & $40-85$ & $<0.01$ & 78 & $53-90$ & $<.0001$ & 75 & $43-89$ & $<.01$ \\
\hline Enhanced & 8,046 & 10 & 4,687 & 6 & 64 & 4 & $-165-65$ & 0.94 & -11 & $\begin{array}{c}-219- \\
61\end{array}$ & 0.85 & -101 & $-558-39$ & \begin{tabular}{|l|}
0.25 \\
\end{tabular} & -100 & $-558-39$ & .25 \\
\hline Overall & 21,380 & 28 & 20,437 & 67 & 380 & 60 & $35-76$ & $<.01$ & 44 & $11-68$ & 0.01 & 52 & $15-72$ & 0.0114 & 44 & $-7-70$ & .08 \\
\hline
\end{tabular}

* For the measures of protection, P1 was the individuals from the higher vaccine coverage clusters and P2 was the individuals from the lowest vaccine coverage clusters as explained in the text.

${ }^{\dagger}$ Cox proportional hazard model was used.

¥ Conditional logistic model was used.

$\S$ Variables selected as covariates were age (at $2^{\text {nd }}$ dose for vaccine/placebo recipients and median date of $1^{\text {st }}$ dose for no vaccine or placebo recipients), individuals living in a household owning at least one luxury item, distance from the household to the nearest health clinic. We did not adjust for any variables for evaluating the enhanced measure of protection due to an insufficient number of outcomes. 